\title{
DESIGN OF FUZZY SELF TUNING PID LOAD FREQUENCY CONTROLLER FOR THE EGYPTIAN POWER SYSTEM
}

\author{
M. A. Abdel Ghany ${ }^{1}$, M. E. Bahgat ${ }^{2}$, W. M. Refaey ${ }^{2}$ and F. N. Hassan ${ }^{2}$ \\ ${ }^{1}$ Faculty of Computer science-Nahda University (NUB), Bani Sweif City, Egypt \\ ${ }^{2}$ Electrical Power \& Machines Department, Faculty of Engineering - Helwan, \\ University of Helwan, Cairo, Egypt
}

\begin{abstract}
In this paper, a fuzzy self tuning is designed to update the proportional, integral, and derivative PID (FST-PID) gains which are optimized based on the Ant Colony System ACS. The design procedure is performed through two steps. Firstly, the PID gains are obtained using ACS algorithm and denoted by ACS-PID. Secondly, a fuzzy self-tuning is designed to self tune such gains. The developed approach is applied to the load-frequency control LFC of the Egyptian power system EPS as a single area with multi unit power system. The studied power system comprises three power plants; non-reheat, reheat, and hydro generation plants. The proposed PID controller is designed on an average point of four loading conditions of the EPS during summer and winter of 2008. The effectiveness of the FST-PID controller in the presence of the system inherent nonlinearity generation rate constraint GRC and uncertain system parameters under different disturbances locations are carried out using MATLABSIMULINK simulation program. The results prove that the proposed method is very useful in self tuning the PID controller gains for the LFC Egyptian Power System.
\end{abstract}

\section{INTRODUCTION}

The power system load frequency control LFC problems are that of sudden small load perturbations which continuously disturb the normal operation of a power system. Many investigations have been done in the area of LFC. Among various types of LFC, the PI/PID controller is most widely applied [1-3]. The PID controllers have been well developed and applied for about half a century [4], and are extensively used for industrial automation and process control today. The main reason is due to their simplicity of operation, ease of design, inexpensive maintenance, low cost, and effectiveness for most linear systems. The PID controller usually based on linearized models of the nonlinear plants. Consequently, the linearized model parameters will be varied according to the operating condition change. It means that, the PID controller gains which designed at certain operating condition gives an optimal response at that condition but it gives suboptimal response at the other operation conditions. To overcome these drawbacks and to ensure an optimal response of the plant at all operating condition a self tuning of PID controller should be facilitated. Numerous researchers have worked to develop methods to tune these gains. Ziegler-Nichols $(\mathrm{Z} / \mathrm{N})$ is probably the most widely known tuning method [5]. This tuning method is very simple, but is not guaranteed to be always effective because of the changes that may happen to the operating conditions during the process run time. Also, human control is very dependent on an operator's experience and qualification, and as a result many PID controllers are poorly tuned in practice. In recent years, fuzzy sets and fuzzy logic have been considered as effective tools to design fuzzy self tuning of PID controller gains [6]. The goal of fuzzy self tuning PID controller design is to update the controller gains $\mathrm{kp}$, ki, and $\mathrm{kd}$ to meet a given set of closed loop system performance requirements. 
Several control techniques based on Fuzzy and Takagi-Sugeno TS fuzzy control system theory have been applied to LFC power system as promising new tools to improve the controller performances [7].

In this paper, the design steps of fuzzy self-tuning PID controller are proposed through two parts. The first one deals with optimal PID controller gains that obtained based on the Ant Colony System ACS. The latter, is a fuzzy logic control FLC part, which has self tuning capabilities. In this case, the proportional, integral and derivate $\mathrm{kp}, \mathrm{ki}$, and $\mathrm{kd}$ gains in a system can be self-tuned on-line with the output of the system. The developed controller is applied on four different loading conditions of the Egyptian Power System EPS load frequency control during the summer and winter of 2008 [8-11]. The robustness of the EPS is ensured when the proposed controller is applied on these four loading conditions in the presence of the system nonlinearity generation rate constraint GRC and uncertain system parameters. The simulation results using MATLAB/ Simulink Toolbox are carried out with the power system subjected to disturbances variation and in the presence of the system inherent nonlinearity. Simulation comparison is made between the fuzzy self tuning of PID (FSTPID) obtained from ACS and ACS PID controllers introduced in [2]. The results prove that the fuzzy logic method is very useful in tuning the PID controller parameters for the LFC Egyptian Power System.

\section{THE EGYPTIAN LFC SYSTEM}

The EPS's total installed generation capacity and peak load were, respectively, about 22500 MW and 19740 MW in 2008 [8-11]. The EPS was incorporating about 180 generating units belonging to the following categories:

1- Nonreheat generating units represented by gas turbine power stations and few steam power stations which is about $24 \%$ of the installed capacity.

2- Reheat generating units represented by the majority of thermal stations and combined cycle power stations which is about $62 \%$ of the installed capacity.

3- Hydro electric power stations which is about $14 \%$ of the installed capacity.

The national energy control center NECC of the Egyptian Electricity Authority has developed a dynamic power frequency model for the EPS [8-11]. The NECC model is originally built for solving load shedding problems and considers only the effect of primary reserve. The EPS consists of seven strongly tied zones. These zones have not considered any interconnection details and the study is carried out based on single area power system model [8-11]. The validity of the model has been tested through two different generator outage contingencies in two different loading conditions [11].

Fig. (1) - displayed in the appendix - shows the block diagram of the Egyptian Power System LFC model as represented by Simulink $[2,8,9]$. The parameters of this model are divided into two sets. The first set of parameters does not depend on system operating conditions. Table (1) lists the values of those parameters as estimated by NECC [8-11]. The other set of parameters changes with time according to the operating condition. The data required to calculate the changing parameters are concerned with the data of each generator including: status (ON or OFF), type of unit (nonreheat, reheat, or hydro), unit rating (MW), unit output (MW) for the operating condition under study, inertia of the unit, and the spinning reserve of the unit in percentage of the unit rating.

The Simulink model considers the generating rate constraints GRC for different generating units. The applied values for GRC are $0.1 \mathrm{p} . \mathrm{u} \mathrm{MW} / \mathrm{min}$ and $0.2 \mathrm{p} . \mathrm{u} \mathrm{MW} / \mathrm{min}$ for reheat turbines and nonreheat turbines, respectively. The GRC of hydro plants is neglected since its actual value is much greater corresponding to the time durations of practical disturbances [8, 9]. 
Table (1) - Egyptian LFC Parameters

\begin{tabular}{|c|c|c|c|c|c|}
\hline Parameter & value & Parameter & value & Parameter & value \\
\hline $\mathrm{D}$ & 0.028 & $\mathrm{R}_{1}$ & 2.5 & $\mathrm{~T}_{\mathrm{w}}$ & 1.0 \\
\hline $\mathrm{T}_{1}$ & 0.4 & $\mathrm{R}_{2}$ & 2.5 & $\mathrm{R}_{\mathrm{L}}$ & 0.8 \\
\hline $\mathrm{T}_{2}$ & 0.4 & $\mathrm{R}_{3}$ & 1.0 & $\mathrm{~T}_{\mathrm{L}}$ & 2.5 \\
\hline $\mathrm{T}_{\mathrm{b}}$ & 6 & $\mathrm{~T}_{\mathrm{d}}$ & 5 & & \\
\hline $\mathrm{m}$ & 0.5 & $\mathrm{~T}_{3}$ & 90 & & \\
\hline
\end{tabular}

The dynamic equations of this model can be written in the state-space form as : where:

$$
\dot{\mathbf{x}}(\mathbf{t})=\mathbf{A} \mathbf{x}(\mathbf{t})+\mathbf{B} \mathbf{u}(\mathbf{t})
$$

$\mathrm{x}(\mathrm{t})=\left[\begin{array}{llll}\Delta \mathrm{F}(\mathrm{t}) & \Delta \mathrm{P}_{1}(\mathrm{t}) \quad \Delta \mathrm{P}_{2}(\mathrm{t}) \quad \Delta \mathrm{V}_{2} \quad \Delta \mathrm{P}_{3}(\mathrm{t}) \quad \Delta \mathrm{G}(\mathrm{t})\end{array}\right]^{\mathrm{t}}$

$\Delta \mathrm{F}(\mathrm{t})=\mathrm{x}_{1}(\mathrm{t})=$ the incremental frequency deviation Hz.

$\Delta \mathrm{P}_{1}(\mathrm{t})=\mathrm{x}_{2}(\mathrm{t})=$ incremental change in non-reheat plant output in p.u MW.

$\Delta \mathrm{P}_{2}(\mathrm{t})=\mathrm{x}_{3}(\mathrm{t})=$ incremental change in reheat plant output in p.u MW.

$\Delta \mathrm{V}_{2}=\mathrm{x}_{4}(\mathrm{t})=$ incremental opening in steam valve of reheat plant output in p.u MW.

$\Delta \mathrm{P}_{3}(\mathrm{t})=\mathrm{x}_{5}(\mathrm{t})=$ incremental change in hydro plant output in p.u MW.

$\Delta \mathrm{G}(\mathrm{t})=\mathrm{x}_{6}(\mathrm{t})=$ incremental opening in hydro plant inlet vane in p.u MW.

The system model matrices $\mathbf{A} \boldsymbol{\&} \mathbf{B}$ of the EPS are given in $[2,8,9]$

Four loading conditions of the EPS are considered to design the BF-based PID gains. These four loading conditions represent the max and min loads in two daily load curves of the EPS in 2008 [8-11], which are the two days of the max water discharge Monday 30/6/2008 and the min water discharge Tuesday 8/1/2008 from hydro power plants. Four MATLAB mfiles are used to calculate the changing parameters of the Simulink model. Each $\mathrm{m}$-file contains 6 data sets for each of the 180 generating units installed at 2008. The first one indicates whether the unit is ON or OFF, while the second one is for the generating unit type. The third and the fourth are for the rating and operating MW of the unit. The fifth and the sixth are for the unit inertia and the unit reserve in percent of its rating. Table (2) shows the calculated parameters out of the four $\mathrm{m}$-files for the four considered operating conditions.

Table (2) - Operating conditions of EPS at 2008

\begin{tabular}{|c|c|c|c|c|}
\hline & $\mathrm{H}$ & $\mathrm{Pn}_{1}$ & $\mathrm{Pn}_{2}$ & $\mathrm{Pn}_{3}$ \\
\hline Con1 & 5.7096 & 0.2529 & 0.6107 & 0.1364 \\
\hline Con2 & 6.0168 & 0.3002 & 0.5200 & 0.1798 \\
\hline Con3 & 5.8552 & 0.2433 & 0.6179 & 0.1389 \\
\hline Con4 & 6.1452 & 0.3335 & 0.5455 & 0.1210 \\
\hline
\end{tabular}

The average point of four loading conditions can be obtained from Table 2 .

\section{FUZZY SELF TUNING OF PID DESIGN PROCEDURE}

The proposed design procedure includes two steps:

1. Find the optimal gains of PID which are controlled the system [6].

2. Design Fuzzy logic control (FLC) part, which has self tuning capabilities for the obtained PID.

\section{A. Optimal PID controller}

The transfer function of the PID controller given in (2) : 


$$
\mathbf{k}(\mathbf{s})=\mathbf{k}_{\mathbf{p}}+\frac{\mathbf{k}_{\mathbf{i}}}{\mathbf{s}}+\mathbf{k}_{\mathbf{d}} \mathbf{s}
$$

where: $\mathrm{k}_{\mathrm{p}}, \mathrm{k}_{\mathrm{i}}$ and $\mathrm{k}_{\mathrm{d}}$ are proportional, integral and differential gains respectively. The input to the controller is the incremental frequency deviation $\Delta \mathrm{F}$ and the output is $\mathrm{u}(\mathrm{s})$ as mentioned in Eq. (1).

$$
\mathbf{u}(\mathbf{s})=-\mathbf{k}(\mathbf{s}) * \Delta \mathbf{F}
$$

In this study, the optimal PID are based on the Ant Colony System ACS optimization algorithms which were given and discussed in [2,12]. The results of the optimal PID gains of the Egyptian LFC System are tabulated in Table. (3)

Table (3) - PID Controller Gains

\begin{tabular}{|c|c|c|c|}
\hline & $\mathbf{k}_{\mathbf{p}}$ & $\mathbf{k}_{\mathbf{i}}$ & $\mathbf{k}_{\mathbf{d}}$ \\
\hline ACS & 1.9192 & $\mathbf{0 . 1 5 1 5 2}$ & $\mathbf{0 . 5 5 5 5}$ \\
\hline
\end{tabular}

\section{B. Designs Steps of Fuzzy Self Tuning for the PID Controller}

Figure (2) shows the block diagram of a conventional fuzzy logic system with two inputs and one output variables. The fuzzy logic systems are used as a controller with various control schemes. The most obvious one is shown in Figure (3), where the fuzzy controller is in the forward path in a feedback control system. The process output is compared with a reference, and if there is a deviation, the controller takes action according to the control strategy [13-15].

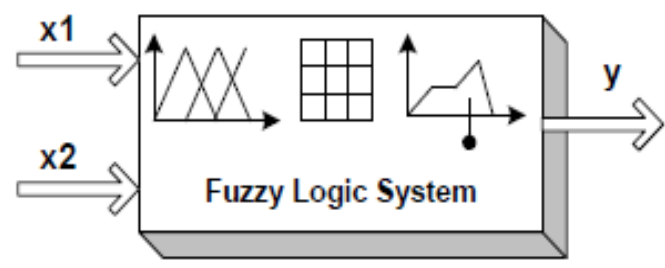

Fig (2) - Fuzzy Logic System

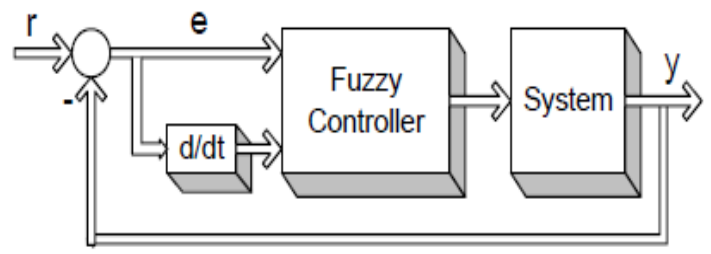

Fig (3) - The Control System Structure with a Fuzzy Controller

The proportional, integral and derivate $\left(\mathrm{K}_{\mathrm{P}}, \mathrm{K}_{\mathrm{I}}, \mathrm{K}_{\mathrm{D}}\right)$ gains in a system can be self-tuned online with the output of the system under control as shown in Fig (4).

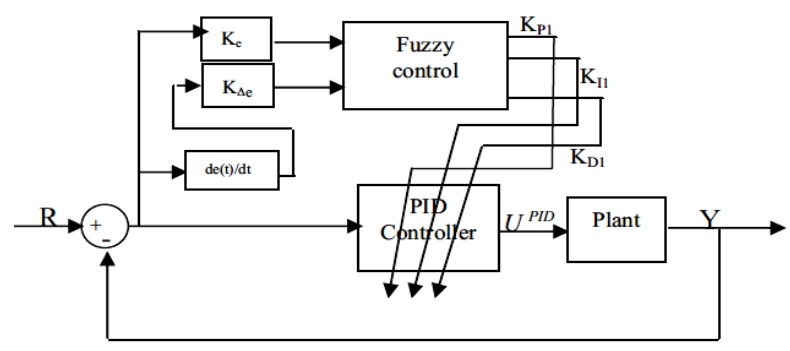

Fig (4) - Fuzzy Self Tune PID controller

The designs steps of fuzzy self tuning can be summarized as follows [6]:

1- Write the PID controller by the following equation:

$$
U=K_{P}+K_{I} \int e d t+K_{D} \frac{d e(t)}{d t}
$$


This equation can be written as :

$$
U=K_{P 2}+K_{I 2} \int e d t+K_{D 2} \frac{d e(t)}{d t}
$$

Where:

$$
\begin{aligned}
& K_{P 2}=K_{P} * K_{P 1}, K_{I 2}=K_{I} * K_{I 1}, K_{D 2}=K_{D} * K_{D 1} \\
& \mathrm{~K}_{\mathrm{p} 1}, \mathrm{~K}_{\mathrm{I} 1}, \mathrm{~K}_{\mathrm{D} 1} \text { are the gain outputs from fuzzy controller }
\end{aligned}
$$

2- Put the input membership functions of e and $\Delta \mathrm{e}$ as linguistic labels shown in Fig (5). The inputs are \{Negative Big, Negative medium, Negative small, Zero, Positive small, Positive medium, Positive Big $\}$, and are referred to in the rules bases as $\{\mathrm{NB}, \mathrm{NM}, \mathrm{NS}, \mathrm{ZE}, \mathrm{PS}$, PM , PB $\}$, and the linguistic labels of the outputs are $\{$ Zero, Medium small, Small, Medium, $\mathrm{Big}$, Medium big, very big $\}$ and referred to in the rules bases as $\{\mathrm{Z}, \mathrm{MS}, \mathrm{S}, \mathrm{M}, \mathrm{B}, \mathrm{MB}$, VB\}. The output membership functions is shown in Fig (6) [6].
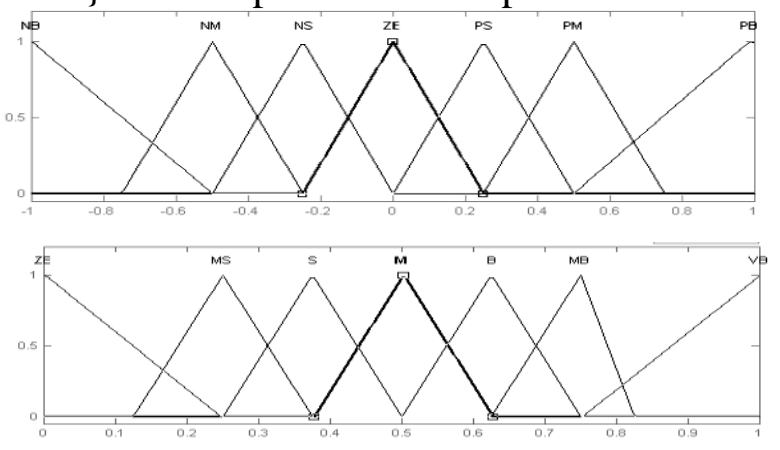

Fig (5) - Input membership functions of e and $\Delta e$,

Fig (6) - Output Membership Functions

Where:

Ke: error input normalizing gain.

$\mathrm{K} \Delta \mathrm{e}: \Delta$ error input normalizing gain.

3- Calculate the values of normalizing gain by dividing the boundary values of the universe of discourse of the input member ship functions of e and $\Delta \mathrm{e}$ by the maximum of the original values of e and $\Delta \mathrm{e}$ respectively. This is to normalize them within the boundaries of the membership universe of discourse.

The rule base for determining $\mathrm{K}_{\mathrm{P} 1}$ is shown in Table (4)

Table (4) - Rule base for determining KP1

\begin{tabular}{|c|c|c|c|c|c|c|c|}
\hline $\mathbf{c e}$ & $\mathbf{N B}$ & $\mathbf{N M}$ & $\mathbf{N S}$ & $\mathbf{Z E}$ & $\mathbf{P S}$ & $\mathbf{P M}$ & $\mathbf{P B}$ \\
\hline $\mathbf{N B}$ & $\mathrm{VB}$ & $\mathrm{VB}$ & $\mathrm{VB}$ & $\mathrm{VB}$ & $\mathrm{VB}$ & $\mathrm{VB}$ & $\mathrm{VB}$ \\
\hline $\mathbf{N M}$ & $\mathrm{MB}$ & $\mathrm{MB}$ & $\mathrm{MB}$ & $\mathrm{MB}$ & $\mathrm{B}$ & $\mathrm{MB}$ & $\mathrm{VB}$ \\
\hline $\mathbf{N S}$ & $\mathrm{B}$ & $\mathrm{B}$ & $\mathrm{B}$ & $\mathrm{B}$ & $\mathrm{MB}$ & $\mathrm{B}$ & $\mathrm{VB}$ \\
\hline $\mathbf{Z E}$ & $\mathrm{ZE}$ & $\mathrm{ZE}$ & $\mathrm{ZE}$ & $\mathrm{MS}$ & $\mathrm{S}$ & $\mathrm{S}$ & $\mathrm{S}$ \\
\hline $\mathbf{P S}$ & $\mathrm{B}$ & $\mathrm{B}$ & $\mathrm{B}$ & $\mathrm{B}$ & $\mathrm{MB}$ & $\mathrm{B}$ & $\mathrm{VB}$ \\
\hline $\mathbf{P M}$ & $\mathrm{MB}$ & $\mathrm{MB}$ & $\mathrm{MB}$ & $\mathrm{MB}$ & $\mathrm{B}$ & $\mathrm{MB}$ & $\mathrm{VB}$ \\
\hline $\mathbf{P B}$ & $\mathrm{VB}$ & $\mathrm{VB}$ & $\mathrm{VB}$ & $\mathrm{VB}$ & $\mathrm{VB}$ & $\mathrm{VB}$ & $\mathrm{VB}$ \\
\hline
\end{tabular}

The rule base for determining $\mathrm{K}_{\mathrm{I} 1}$ is shown in Table (5). 
Table (5) - Rule base for determining $\mathrm{K}_{\mathrm{I1}}$.

\begin{tabular}{|c|c|c|c|c|c|c|c|}
\hline e & NB & NM & NS & ZE & PS & PM & PB \\
\hline NB & M & M & M & M & M & M & M \\
\hline NM & M & M & M & M & M & M & M \\
\hline NS & S & S & S & S & S & S & S \\
\hline ZE & MS & MS & MS & ZE & MS & MS & MS \\
\hline PS & S & S & S & S & S & S & S \\
\hline PM & M & M & M & M & M & M & M \\
\hline PB & M & M & M & M & M & M & M \\
\hline
\end{tabular}

The rule base for determining $K_{D 1}$ is shown in Table (6).

Table (6) - Rule base for determining $K_{D 1}$

\begin{tabular}{|c|c|c|c|c|c|c|c|}
\hline $\mathbf{e}$ & NB & NM & NS & ZE & PS & PM & PB \\
\hline NB & ZE & MS & S & M & MB & B & VB \\
\hline NM & MS & S & M & B & B & B & VB \\
\hline NS & S & M & B & MB & VB & VB & VB \\
\hline ZE & M & B & MB & MB & VB & VB & VB \\
\hline PS & MB & MB & VB & VB & VB & VB & VB \\
\hline PM & B & MB & VB & VB & VB & VB & VB \\
\hline PB & VB & VB & VB & VB & VB & VB & VB \\
\hline
\end{tabular}

4- Apply the defuzzification process as final step to converts the fuzzy output to crisp value to be used as a non fuzzy control action.

5- Choose the most popular defuzification method that is the center of gravity or center of area which is formulated as follows:

$$
\mathbf{u}=\frac{\sum_{\mathrm{i}=1}^{\mathrm{r}} \mathbf{u}\left(\mathbf{u}_{\mathrm{i}}\right) \mathbf{u}_{\mathrm{i}}}{\sum_{\mathrm{i}=1}^{\mathrm{r}} \mathrm{I}\left(\mathrm{u}_{\mathrm{i}}\right)}
$$

Where $u\left(u_{i}\right)$ is the membership grad (weight) of the element $u_{i}$ which is the output of the rule.

Figure (1) shows fuzzy self tuning PID controller for the LFC Egyptian Power System built in MATLAB/ Simulink.

\section{SIMULATION RESULTS}

The simulation results are obtained using MATLAB package and Simulink Toolbox. To be more practical, the Simulink model considers the generating rate constraints GRC for different generating units as described before in [8-11]. The study is started by calculating the optimal values of PID gains using ACS algorithm for the Egyptian Power System load frequency control as a first step. The results of FST-PID gains are obtained as in Table (1). 
The second step deals with fuzzy self tuning design of PID. Several cases are considered for verification of the effectiveness of the proposed FST-PID controllers.

Case 1: Step disturbance $\Delta P_{d}=1 \%$

In order to compare the optimized ACS-PID with the FST-PID a relatively disturbance $\Delta \mathrm{P}_{\mathrm{d}}$ of $1 \%$ is applied to the EPS. The time response of the frequency deviation $\Delta \mathrm{F}$ and the control input are displayed in Fig (3). It is seen that when the FST-PID is used, the damping of the system frequency is improved significantly and settles to the nominal value quickly. In addition, it is clear that FST-PID responds a lot better than ACS-PID, i.e., less overshoots oscillations with acceptable settling time than ACS-PID.
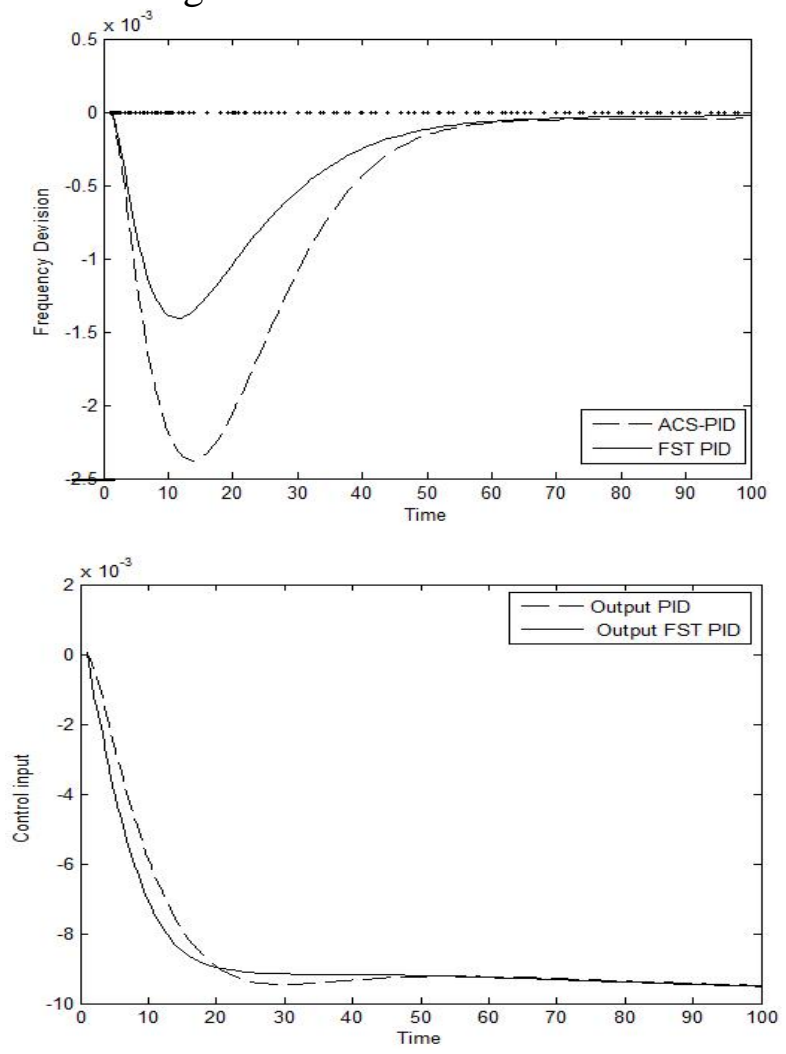

(a) $\Delta \mathrm{F}_{1}$

(b) $\Delta \mathrm{U}$

Fig (3) - System dynamic responses for case 1

\section{Case 2: Disturbance Variations}

Fig (4), illustrates the dynamic response of $\Delta \mathrm{F}$ and $\Delta \mathrm{U}$ when a step of $\Delta \mathrm{P}_{\mathrm{d}}=1 \%$ is applied during $5 \leq \mathrm{t} \leq 30$ seconds and $\Delta \mathrm{P}_{\mathrm{d}}=0 \%$ during $0 \leq \mathrm{t} \leq 5$ seconds. It is observed that the FSTPID gives better response as compared to ACS-PID controller in terms of faster settling time with zero steady-state error. The FST-PID still gives better results than ACS-PID. 

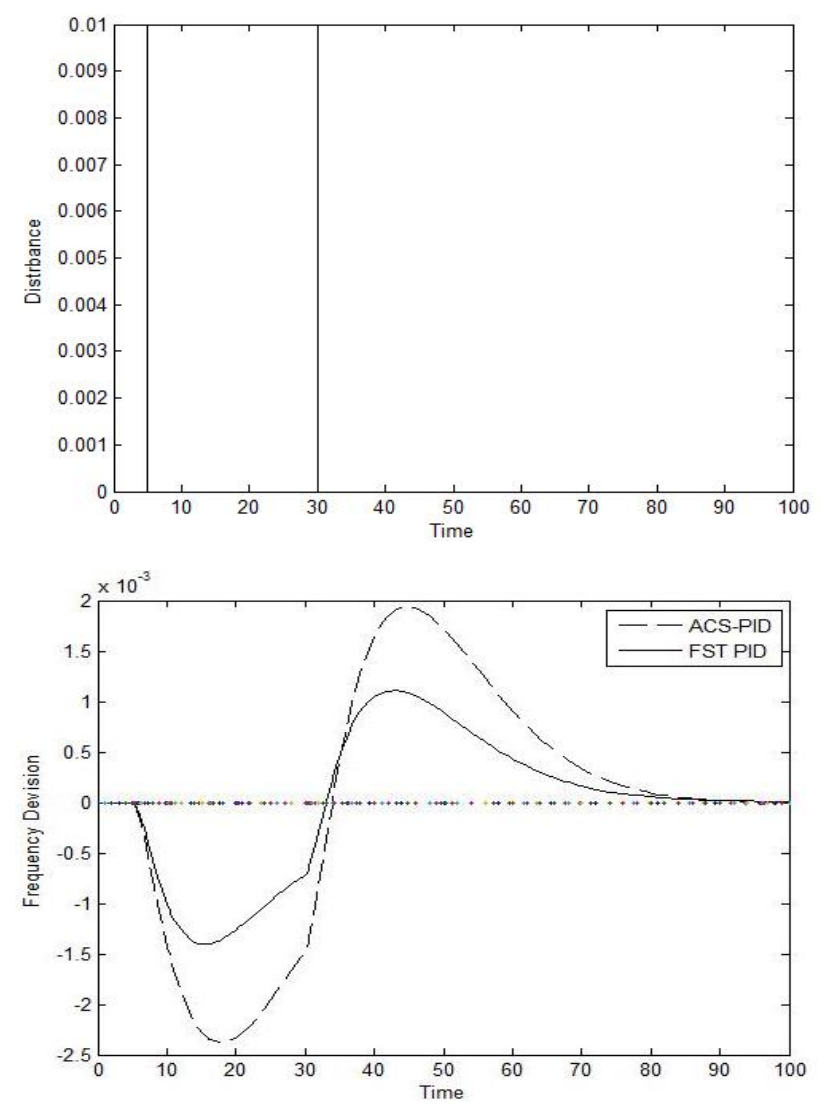

(a) $\Delta \mathbf{P}_{\mathbf{d}}$

(b) $\Delta \mathbf{F}$

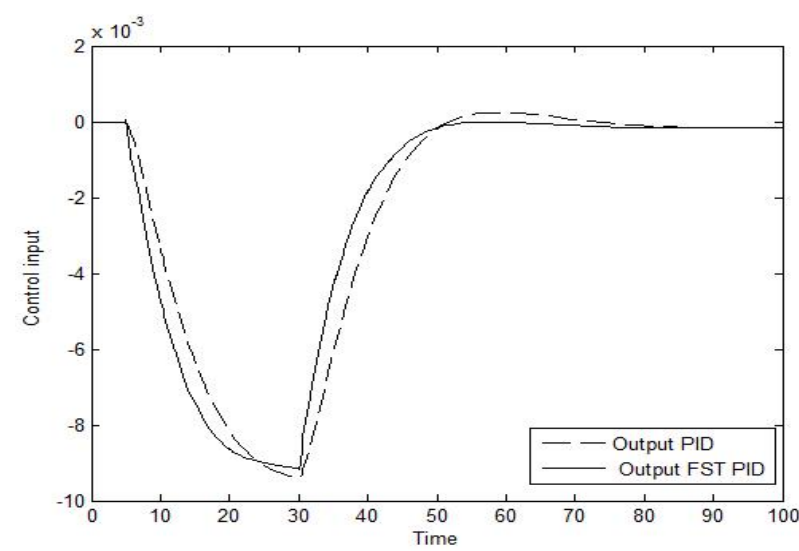

(c) $\Delta \mathrm{U}$

Fig (4) - System dynamic responses for case 2

\section{Case 3: Tracking Disturbance Variations}

Fig (5) and (6) show the dynamic response of $\Delta \mathrm{F}$ and $\Delta \mathrm{U}$ following a variation of $\Delta \mathrm{P}_{\mathrm{d}}$ as seen in Fig (5) - (a). and Fig (6) - (a). This variation covers both tracking represented by the ramp and regulation which represented by a step change. It is clear that the FST-PID gives better results than ACS-PID while keeping the system stability and responds effectively within an acceptable period of time before arriving to zero steady-state. Also, Fig (6) shows better responses from FST-PID against the ACS-PID. 
DESIGN OF FUZZY SELF TUNING PID LOAD FREQUENCY CONTROLLER FOR THE EGYPTIAN POWER SYSTEM
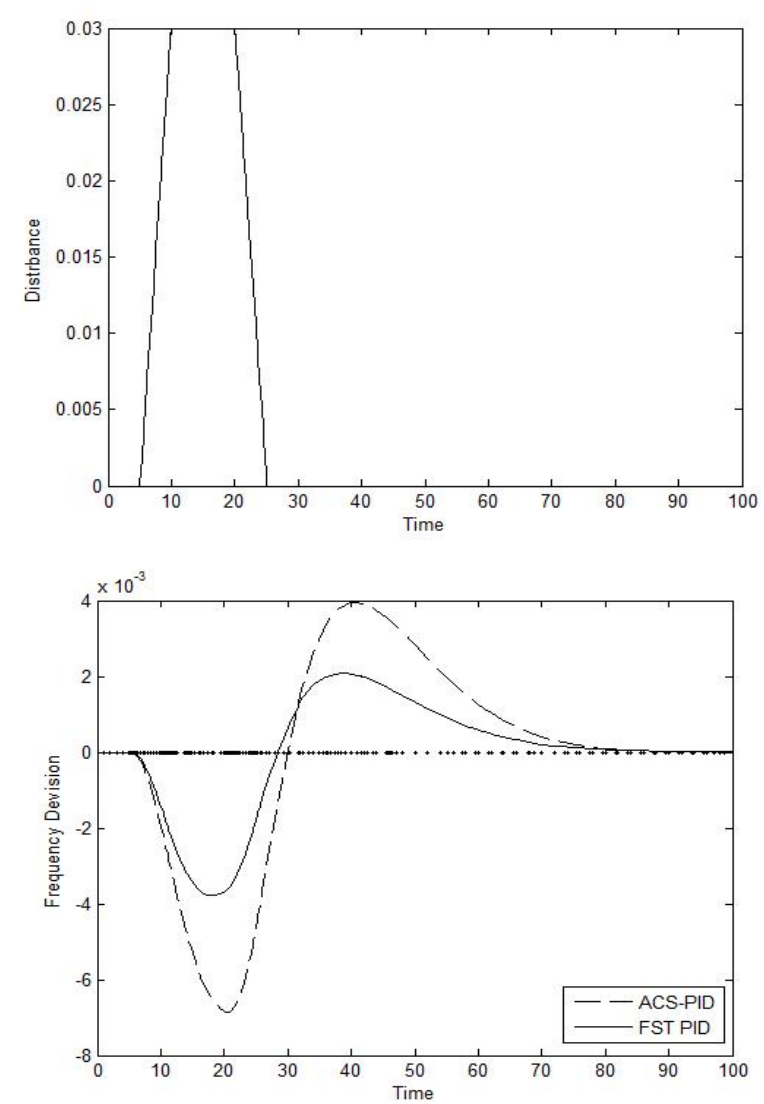

(a) $\Delta \mathbf{P}_{\mathbf{d}}$

(b) $\Delta \mathbf{F}$

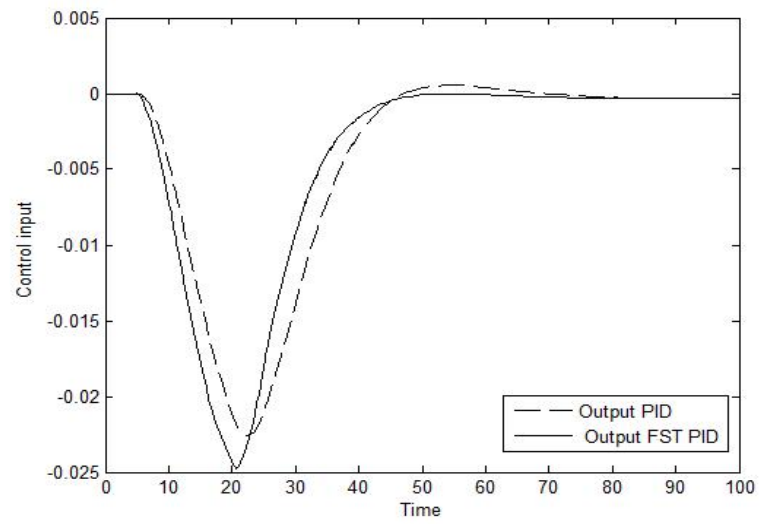

(c) $\Delta \mathrm{U}$

Fig (5) - System dynamic responses for case 3 


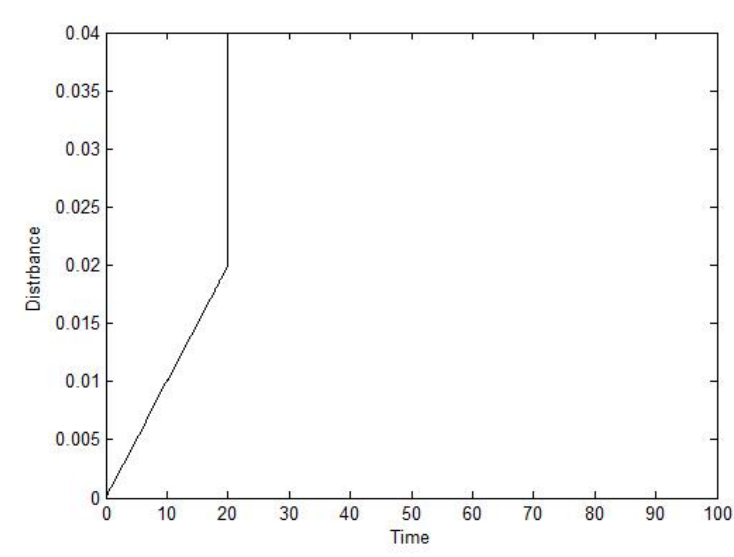

(a) $\Delta \mathbf{P}_{\mathbf{d}}$

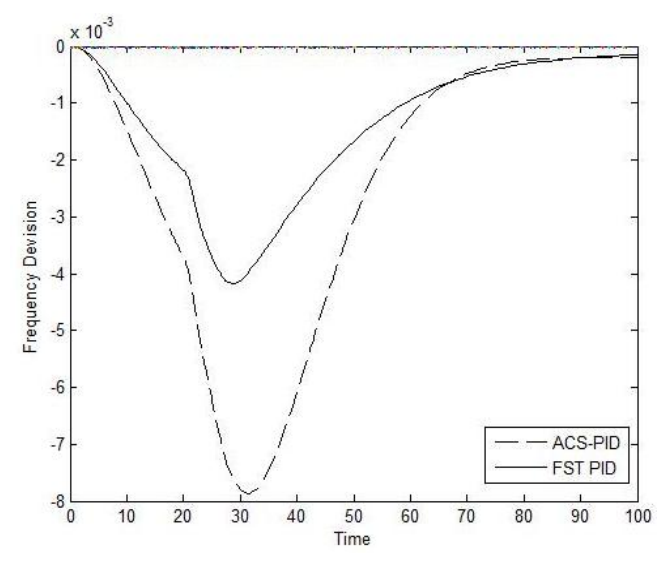

(b) $\Delta \mathbf{F}$

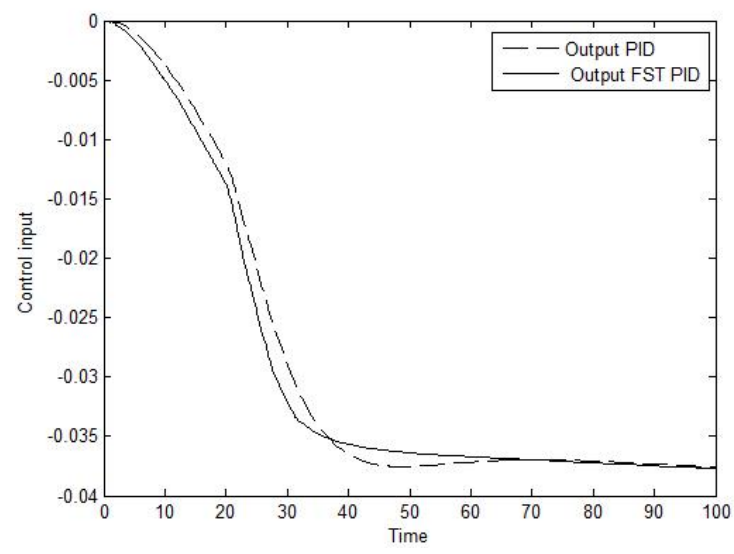

(c) $\Delta \mathrm{U}$

Fig (6) - System dynamic responses for case 3

Case 4: Uncertain System parameters at $\square \mathbf{P}_{\mathbf{d}}=1 \%$

The parameters $D, R_{1}, R_{2}, R_{3}, T_{1}, T_{2}, T_{3}, T_{w}, T_{h}$ and $T_{d}$ are taken into account as uncertain parameters with $\pm 40 \%$ of EPS power system. In addition, GRC is assumed.

The dynamic performances of the FST-PID and ACS-PID controllers under uncertain parameters when the step input disturbance of $\Delta \mathrm{P}_{\mathrm{d}}=1 \%$ is applied to the system is applied to EPS is shown in Fig (7). Results obtained by the FST-PID are very encouraging in the presence of system nonlinearity and uncertain parameters than ACS-PID. 

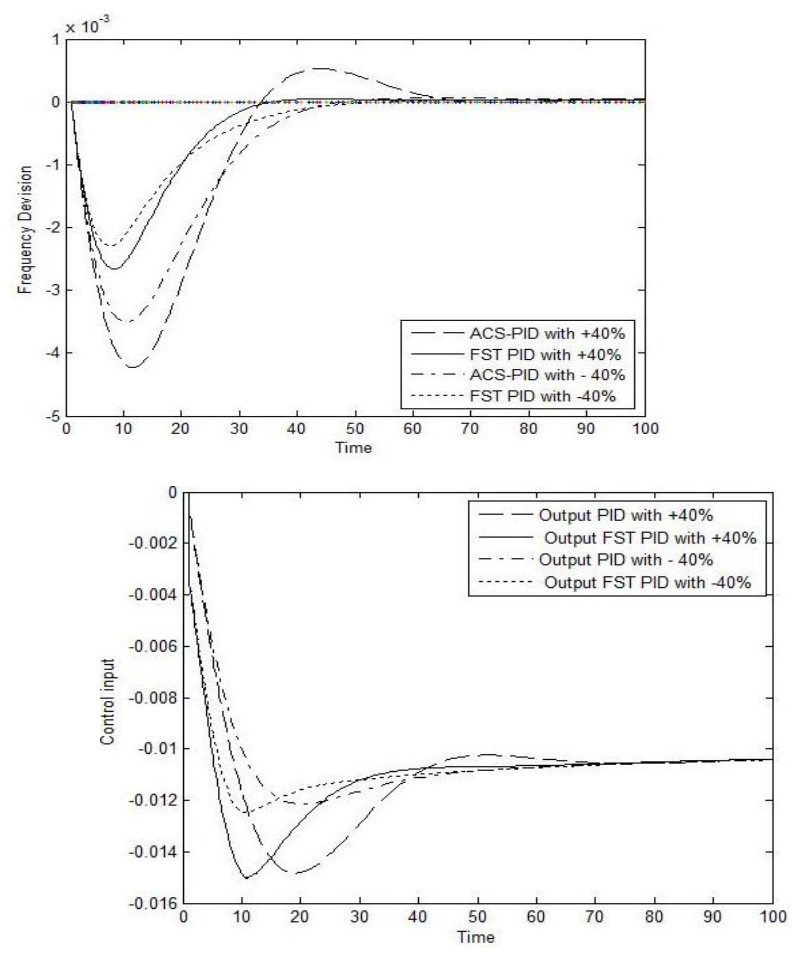

(a) $\Delta \mathbf{F}_{1}$

(b) $\Delta \mathbf{U}$

\section{CONCLUSION}

Fig (7) - System dynamic responses for case 4

This paper has addressed and compared the performance of fuzzy self tuning of PID and ACS-PID controllers for the Egyptian power system EPS as a single area with multi unit power system. The design steps of the proposed FST-PID are presented and discussed. The effectiveness of the FST-PID controller in the presence of the system inherent nonlinearity generation rate constraint GRC and uncertain system parameters under different disturbances locations are performed. The results have shown that, for the FST-PID proposed in this paper, the dynamic performance seems to perform better, even when different disturbances are considered. The better performance (in terms of a fewest oscillations and fastest removing steady state values) of the considered power system when compared with ACS-PID. The results show that the FST-PID controller is very powerful in reducing the frequency deviations under a variety of load perturbations.

\section{REFERENCES}

[1] O. I. Elgerd, Electric Energy System Theory, An Introduction, McGraw-Hill, 1982.

[2] M. A. Abdel Ghany, M. E. Bahgat, W. M. Refaey and F. N. Hassan "Ant Colony Optimum Tuning of PID Load Frequency Controller for the Egyptian Power System", Sixteenth International Middle East Power Systems Conference (MEPCON'14), Ain Shams University, Egypt, 2014.

[3] M. A. Abdel Ghany, M. E. Bahgat, W. M. Refaey, F. N. Hassan" Design of Fuzzy PID Load Frequency Controller Tuned by Relative Rate Observer for the Egyptian Power System " 9th International Conference on Electrical Engineering ICEENG-9, Cairo, Egypt, May 27-29, 2014

[4] G.J. Silva, A. Datta, et al., "New results on the synthesis of PID controllers," IEEE Transactions on Automatic Control, 2002, 47(2), pp. 241-252.

[5] A. M. Abdel Ghany, O. H. Abdalla, PID Load-frequency Controller For a Hydro-thermal Power System, Fourth Middle East Power System Conference MEPCON, PP. 235-239, January 1996.

[6] Maher M.F. Algreer and Yhya R.M. Kuraz "Design Fuzzy Self Tuning of PID Controller for Chopper-Fed DC Motor Drive", Al-Rafidain Engineering Journal, Vol. 16, No. 2, pp. 54-66 A, 2008. 
[7] H.A. Shayanfar, H. Shayeghi and A. Jalili" Takagi-Sugeno Fuzzy Parallel Distribution Compensation Based Three-Area LFC Design" International Journal on "Technical and Physical Problems of Engineering”(IJTPE) September 2011 Issue 8 Volume 3 Number 3 Pages 55-64

[8] Mostafa H. E. Artificial Intelligence-Based Load Frequency Control of Multi-Area Power Systems. Ph.D. Thesis, faculty of engineering, Ain Shams university, Cairo, Egypt, 1999

[9] Abdel Ghany M. A. Artificial Intelligent and Static Output Feedback Controllers for Power System Load Frequency Control. MS.c. Thesis, Faculty of Engineering, Helwan University, Helwan, Egypt, 2014.

[10] Egyptian Electricity Holding Company, 2007/2008 Annual Report. http://www.egelec.com/annual\%20report/2007.htm.

[11] Yassin K., Abd-Raboh E., and Al-Domany M.S. Fast Power System Restoration Via Load Shedding Practices in Egyptian Power System. Mansoura Eng. Journal, Vol. 17, pp. 120, Mar Egypt, 1992.

[12] M. Omar, M. Soliman, A. M. Abdel Ghany, and F. Bendary " Ant Colony Optimization based PID for single Area Load Frequency Control" Accepted to be published in ICMIC Conference September 2013.

[13] G.R.Chen and T.T.Pham, "Introduction to fuzzy sets, fuzzy logic, fuzzy control system", CRC.Press,Boac Raton,FL, USA, 2000.

[14] L. Zheng, "A Practical Guide to Tune of Proportional and Integral (PI) Like Fuzzy Controllers," IEEE 1992.

[15] O. Karakal, E. Yesil, M. G“U Zelkaya, and I. Eksin, "Implementation of a New SelfTuning Fuzzy PID Controller on PLC", Turk Journal of Elec. Eng., VoL.13, No. 2, pp. 277-286, 2005

\section{Appendix}

The following figure illustrates the block diagram of the Egyptian Power System LFC model in Simulink representation

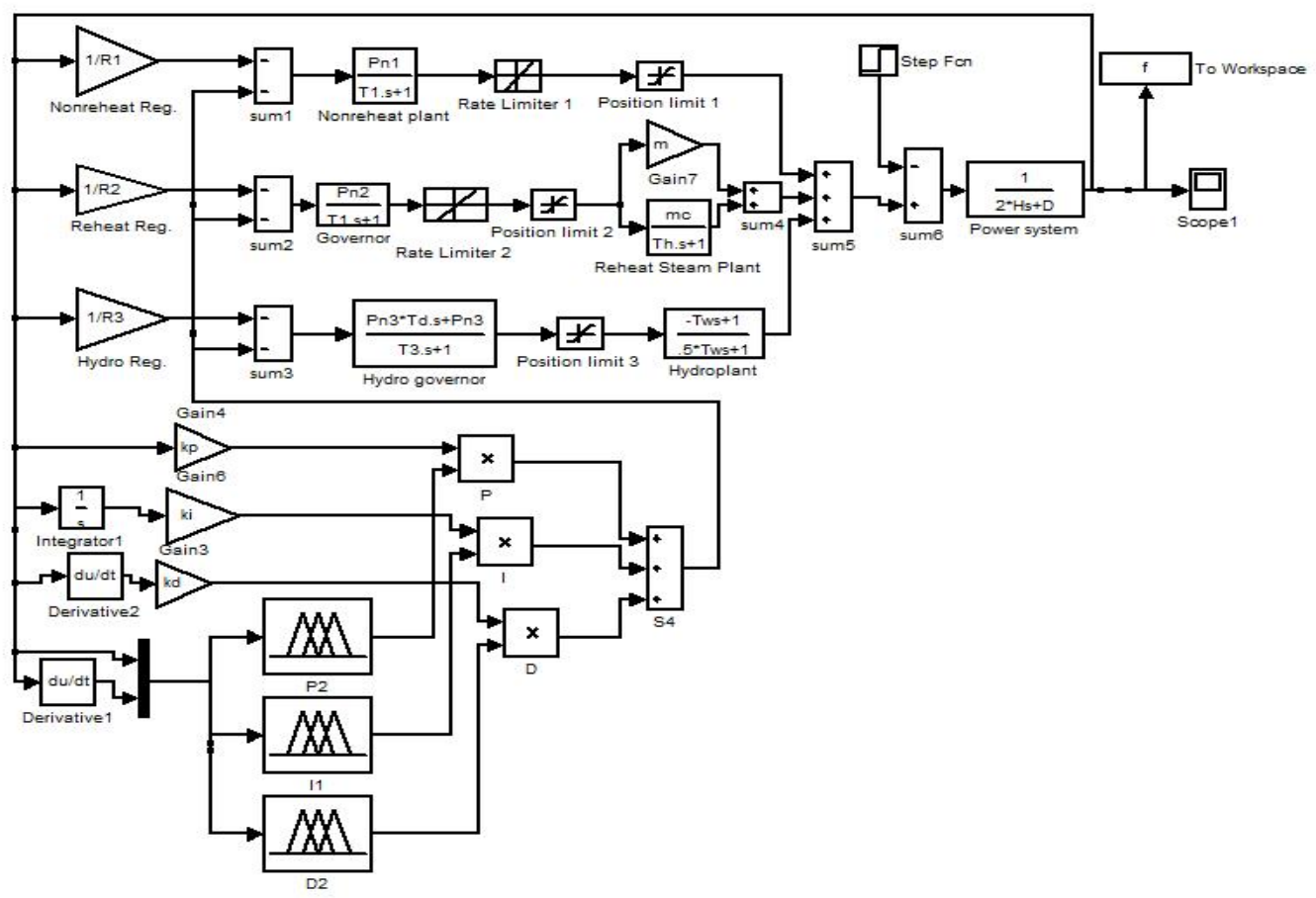

Fig (1) - The block diagram of the Egyptian Power System LFC model in Simulink

\section{List of Symbols}

$\Delta \mathrm{f}$

$\Delta \mathrm{Pd}$
Incremental frequency deviation $(\mathrm{Hz})$

Load disturbance (p.u MW) 
Pn1, Pn2, Pn3 Nominal rated regulating power output for non-reheat, reheat and hydro plants respectively (p.u MW)

$\Delta \mathrm{P} 1, \Delta \mathrm{P} 2, \Delta \mathrm{P} 3 \quad$ Incremental output power at the given loading condition of non-reheat, reheat, and hydro plants respectively (p.u MW)

R1, R2 Average governor droop setting of non-reheat and reheat plants respectively

R3

$\mathrm{H}$

RL

TL

$\mathrm{D}$

T1, T2

(s)

Th

T3

$\mathrm{Tg}$

Tw

$\mathrm{m}$
(Hz/p.u MW)

Permanent droop of the hydro plant speed governor (Hz/p.u MW)

Equivalent inertia const. of the system (s) [19]

Percentage of rotating load

Rotating inertia in $\mathrm{sec} / \mathrm{Hz}$

Load dependency on frequency (p.u MW/Hz)

Steam valve time const. of non-reheat and reheat thermal plant respectively

Time const. of reheat plant (s)

Equivalent time const. of hydro plant (s)

Response time of speed governor (s)

Water starting time in the hydro intake (s)

Fraction of power in high pressure section of Turbine 